\title{
Notch-1 regulates proliferation and differentiation of human bladder cancer cell lines by inhibiting expression of Krüppel-like factor 4
}

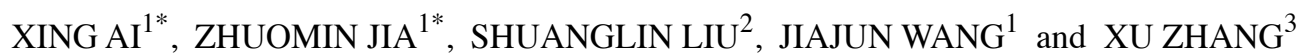 \\ ${ }^{1}$ Department of Urology, Military General Hospital of Beijing PLA, Beijing 100700; \\ ${ }^{2}$ Department of Urology, Wuhan First Hospital, Wuhan, Hubei 430000; \\ ${ }^{3}$ Department of Urology, Chinese PLA General Hospital, Beijing 100853, P.R. China
}

Received March 26, 2014; Accepted June 24, 2014

DOI: $10.3892 /$ or.2014.3350

\begin{abstract}
Inhibition of Notch signaling pathways, consisting of 4 highly conserved receptors (Notch 1-4), induces expression of Krüppel-like transcription factors (KLFs) linked to bladder cancer tumorigenesis and metastasis. Effects of Notch-1 knockdown on cell proliferation, differentiation and KLF4 levels in bladder cancer cell lines were investigated. PsiRNA1-mediated Notch-1 and KLF4 knockdown models and control model without the psiRNA1 vector were constructed using bladder cancer cell lines T24 and BIU87. Cell proliferation, apoptosis and expression of Notch-1 and KLF4 were assessed using 3-(4,5-dimethylthiazol2-yl)-2,5-diphenyltetrazolium bromide (MTT) assay, flow cytometry assay with Annexin V-FITC/PI staining, and reverse transcriptase polymerase chain reaction (RT-PCR) and western blot analysis, respectively. Proliferation was assessed in Notch-1 and/or KLF4 knockdown. The results showed that Notch-1 mRNA and protein expression levels were significantly lower following psiRNA1 vector transfection in both cell lines $(\mathrm{P}<0.05)$. Growth and proliferation of both cell lines were significantly inhibited by Notch-1 knockdown $(\mathrm{P}<0.05)$, and more $\mathrm{G} 0 / \mathrm{G} 1$ arrest and apoptosis were observed compared to those in the control groups $(\mathrm{P}<0.05)$. The effects were time-dependent, peaking between $24-48 \mathrm{~h}$ and declining by $72 \mathrm{~h}$. KLF4 expression was significantly higher in the Notch-1 knockdown group than in control cells $(\mathrm{P}<0.05)$. Notch-1 knockdown cell proliferation was significantly lower than that of Notch-1 and KLF4 knockdown $(\mathrm{P}<0.05)$. In conclusion, Notch-1 may act as an oncogene, regulating the proliferation
\end{abstract}

Correspondence to: Dr Xing Ai, Department of Urology, Military General Hospital of Beijing PLA, Beijing 100700, P.R. China

E-mail: aixing0007@163.com; aixingmedsci@163.com

*Contributed equally

Key words: notch-1, Krüppel-like transcription factor, bladder cancer, proliferation, differentiation and differentiation of bladder cancer cells by inhibiting KLF4. Pending further exploration of pathway variations and crosstalk, these pathways may be useful targets for bladder cancer therapy.

\section{Introduction}

Bladder cancer is a heterogeneous disease that can manifest as superficial tumors (70\% of patients) or muscle-invasive tumors (30\% of patients) with a high risk of invasive metastasis (1). The disease is more common in men, and risk peaks at the ages of 50-70 (2). While high-grade non-invasive tumors can be treated intravesically, the standard of care for muscle-invasive tumors is radical cystoprostatectomy or, alternatively, transurethral tumor resection, radiation and chemotherapy (1). Environmental factors, such as cigarette smoking (1), and genetic factors have been linked to bladder cancer, including oncogenes TP63 (3), epidermal growth factor receptors (EGFR) (4), tumor suppressor genes TP53 and RB1 (5), Ras p21 proteins (6), and cell cycle regulatory proteins, such as CABLES, Ki67 and cyclin D1 (1). Despite these advances, the morbidity associated with invasive bladder cancer remains high (7). Thus, a better understanding of the cell proliferation and differentiation mechanisms in bladder cancer cells is required before improved therapies can be designed.

RNA interference (RNAi) is an endogenous mechanism in which RNA molecules inhibit post-transcriptional gene expression (8). RNAi has been extensively employed by contemporary researchers to establish experimental knockdown models of mammalian cells using engineered gene-specific small interfering RNAs (siRNAs) (9). To better understand tumorigenesis, RNAi techniques have been used to elucidate the function and full cellular processes involved in complex signaling pathways, such as the Notch signaling pathway (10). The Notch signaling pathway is of particular interest in cancer research as it is evolutionarily conserved in virtually all multi-cellular organisms (11), which generally exhibit 4 types of Notch transmembrane receptors: Notch1, Notch2, Notch 3 and Notch4 (12). As key regulators of intracellular signaling, these Notch receptors are critical to cellular differentiation, proliferation and apoptosis $(13,14)$. 
Notch signaling pathways have been implicated in epithelial-mesenchymal transition (EMT) modulation, tumor angiogenesis processes, and anoikis-resistance of tumor cells that have been linked with metastatic tumor development in osteosarcoma, prostate cancer, breast cancer and melanoma (15). Notch signaling was first implicated in tumorigenesis when the recurrent $\mathrm{t}(7 ; 9)(\mathrm{q} 34 ; \mathrm{q} 34.3)$ chromosomal translocation in the human Notchl gene was identified in a subset of cells from human pre-T-cell acute lymphoblastic leukemia (T-ALL) (16). Since then, Notch signaling has been recognized as a potential target for therapeutic intervention in cancer, although available data pertaining to Notch signaling in bladder cancer is limited.

It has been reported that the Notch cascade directly regulates the expression of intestinal epithelium Krüppel-like transcription factors (KLFs) (17), key regulators of cell proliferation, differentiation and apoptosis. Members of the KLF family are characterized by triplicate C-terminus Cys2 His2 zinc fingers separated by highly conserved H/C links (18). Downregulation of several of the 15 identified KLFs, particularly $K L F 4$, has already been linked to growth suppression and apoptosis induction in bladder cancer (19). While short-chain fatty acids can increase KLF4 expression, Notch signaling acts to inhibit KLF4 expression (20). Thus, Notch inhibition indirectly induces KLF4 expression (20), potentially playing a role in tumorigenesis and metastasis in bladder cancer.

To further characterize the role of Notch-1 in the bladder cancer cells, RNAi was used to generate Notch-1 knockdown models of human bladder cancer cell lines (T24 and BIU87) using siRNA eukaryotic expression vector Notch-1 (psiRNA1). KLF4 expression in bladder cancer cells after transfection was also detected in order to investigate the relationship between Notch-1 and KLF4 levels in bladder cancer cells. This study provides a basis for understanding the role of Notch-1 in cell proliferation and differentiation in bladder cancer.

\section{Materials and methods}

Cell cultures. Human bladder cancer cell lines T24 and BIU87 were provided by our lab. All cells were maintained at $37^{\circ} \mathrm{C}$ in a humidified atmosphere of $5 \% \mathrm{CO}_{2}$ in RPMI-1640 medium supplemented with $10 \%(\mathrm{v} / \mathrm{v})$ heat-inactivated fetal calf serum (FCS; both from Gibco, USA).

Transfection of notch-expressing vectors. The siRNA eukaryotic expression vector of Notch-1 (psiRNA1) was constructed and identified as previously described (21). Then, psiRNA1 was transfected into T24 and BIU87 cells (Notch-1 knockdown cells) using a Lipofectamine ${ }^{\mathrm{TM}} 2000$ Transfection kit (Invitrogen, Netherlands), according to the manufacturer's instructions, using the siRNA sequence: 5'-TGGCGGGAAGTGTGAAGCG-3'. A vector without psiRNA1 was also transfected into T24 and BIU87 cells as control cells. Following transfection, all cells were treated with $800 \mu \mathrm{g} / \mathrm{ml} \mathrm{G} 418$ (Sigma, USA) for 3 weeks. Stably transfected cells (resistant clones) were ring-cloned and cultured for further analysis. Downregulation of KLF4 expression (KLF4 knockdown cells) was achieved using siRNA against human KLF4 and a negative control siRNA was established for these cells.
Cell proliferation assessment by MTT assay. The proliferation of Notch-1 knockdown and KLF4 knockdown cells was assessed simultaneously by 3-(4,5-dimethylthiazol-2-yl)-2,5 diphenyltetrazolium (MTT) assay. Briefly, cell suspensions $\left(1 \times 10^{3}\right.$ cells/ml) of Notch-1 knockdown, KLF4 knockdown, and their respective control cells were transferred to 96-well plates in triplicate. Cells were then incubated for 12, 24, 36, 48, 72 and $96 \mathrm{~h}$. At $4 \mathrm{~h}$ before the end of each incubation period, $20 \mu \mathrm{l}$ of MTT solution ( $5 \mathrm{mg} / \mathrm{ml}$ ) (Sigma, USA) was added. Then, cells were centrifuged at 2,200 $\mathrm{x}$ g for $5 \mathrm{~min}$, the supernatant was removed, $200 \mu \mathrm{l}$ of dimethyl sulfoxide (DMSO) was added, and the optical density (OD) was determined at $492 \mathrm{~nm}$.

Detection of apoptosis by flow cytometry. For flow cytometry, T24 and BIU87 cells were resuspended to $1 \times 10^{5}$ cells $/ \mathrm{ml}$ in phosphate-buffered saline (PBS) solution supplemented with $10 \%$ FCS. Cells were then transfected as previously described and incubated for 3 days at $37^{\circ} \mathrm{C}$ in $5 \% \mathrm{CO}_{2}$. Cells were then washed, resuspended in PBS, dyed with propidium iodide (PI) for $30 \mathrm{~min}$ at room temperature, and analyzed by flow cytometry using a flow cytometer (BD FACSAria ${ }^{\mathrm{TM}}$ III, FACSCalibur; BD Biosciences, USA). Annexin V-FITC/ PI double staining assay was used to detect cell apoptosis. Briefly, after similar transfection and incubation, cells were collected, centrifuged and washed twice with PBS. Binding buffer was then added to each tube, cells were resuspended, and then incubated with $5 \mu \mathrm{l}$ of Annexin V-FITC and $5 \mu \mathrm{l}$ of PI for $15 \mathrm{~min}$ at room temperature under dark conditions. Flow cytometry was conducted within the following 1-h period.

Clonal forming assay. T24 and BIU87 cells were infected with Notch-1 siRNA, cultured for $24 \mathrm{~h}$, and plated in 24-well plates (200 cells/well). Plates were incubated for 14 days in a humidified incubator at $37^{\circ} \mathrm{C}$, and resultant colonies were stained with $0.5 \mathrm{ml}$ of $0.0005 \%$ crystal violet solution for $1 \mathrm{~h}$. Cells in 5 randomly-selected fields from each sample were counted by microscopy using an Olympus Optical Microscope (CX21; Olympus Japan) (x100), and data were reported as means \pm standard deviation (SD) of five measurements.

$R N A$ preparation and semi-quantitative $R T-P C R$. Total-RNA was extracted from $\sim 5 \times 10^{6}$ to $1 \times 10^{7}$ cells using an RNeasy kit (Qiagen, Germany), according to the manufacturer's instructions. Resultant total-RNA was stored at $-80^{\circ} \mathrm{C}$ for use in the following experiments. Semi-quantitative analysis of Notch-1 mRNA expression was completed by multiplex reverse transcription-polymerase chain reaction (RT-PCR), and $\beta$-actin was used as a standard. The primer sequences used were: Notch-1 forward, 5'-CTGCCAGGACCCGGACAA-3' and reverse, 5'-TACCCGGCAACGTCGTCAATAC-3'; $\beta$-actin forward, 5'-CCTGTACGCCAACACAGTGC-3' and reverse, 5'-ATACTCCTGCTTGCTGATCC-3'. Extracted total-RNA samples $(1 \mu \mathrm{g})$ were subjected to reverse transcriptase reactions to produce cDNA using SuperScript ${ }^{\circledR}$ II reverse transcriptase (Invitrogen Life Technologies), primed with oligo(dT). Semiquantitative PCR was then performed using synthesized cDNA templates. cDNA templates $(1 \mu \mathrm{l})$ were amplified with Platinum Taq DNA polymerase (Invitrogen). PCR products were separated by $1 \%$ agarose gel electrophoresis, stained with 


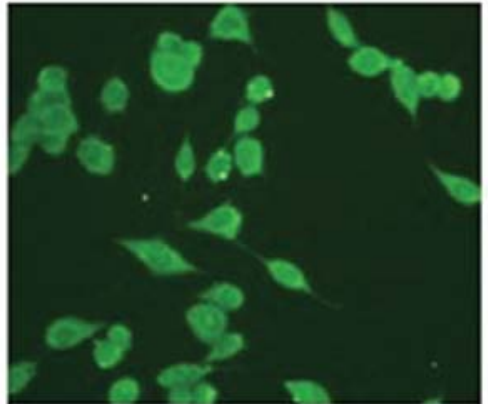

T24:

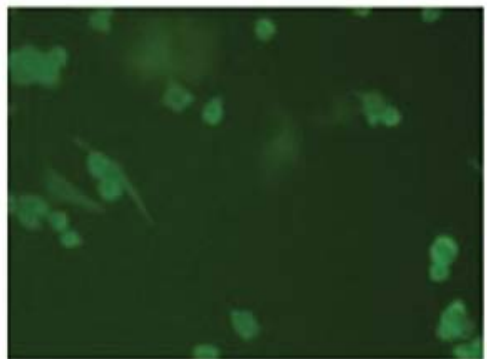

BIU87: $24 \mathrm{~h}$

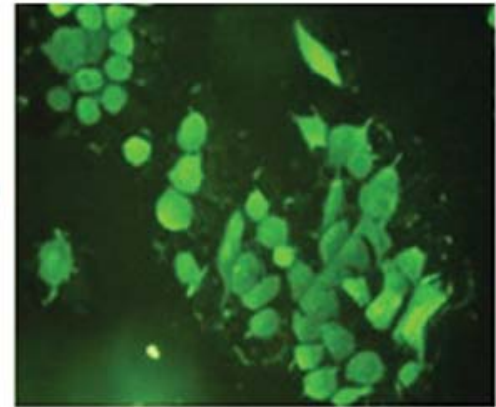

$48 \mathrm{~h}$

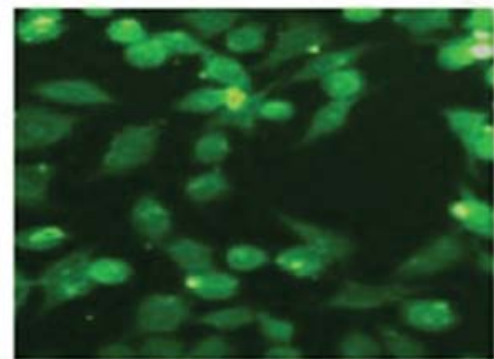

$48 \mathrm{~h}$

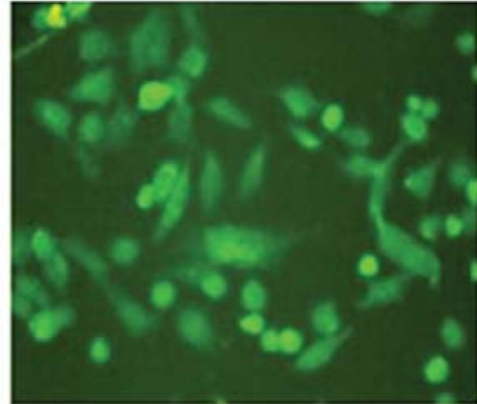

$72 \mathrm{~h}$

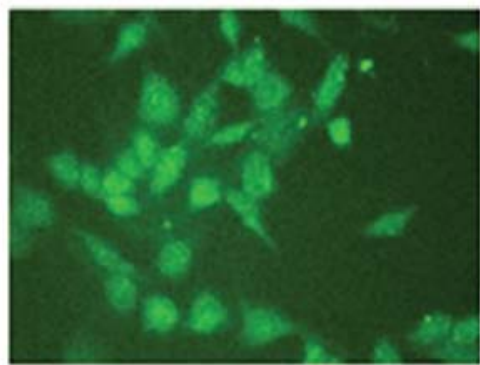

72 h

Figure 1. Transfection efficiency of the T24 and BIU87 cells at 24, 48 and $72 \mathrm{~h}$ after transfection. Transfected cells emitted apparent green fluorescence and non-transfected cells emitted no apparent green fluorescence. Note that the amount of green fluorescence increased with longer incubation time up to $48 \mathrm{~h}$.

ethidium bromide and visualized with an ultraviolet (UV) transilluminator (WUV-L50; Kor).

Quantitative real-time RT-PCR. KLF4 mRNA expression was assessed using quantitative real-time PCR (qRT-PCR) with the QuantiTect SYBR-Green RT-PCR system (Qiagen, Germany), according to the manufacturer-recommended relative quantitation method. After optimization of each primer pair, samples were assayed in a $50 \mu \mathrm{l}$ reaction mixture containing $10 \mu \mathrm{l}$ of sample RNA and optimal concentrations of each primer. Thermal profiles consisted of a $30-\mathrm{min}$ RT step at $50^{\circ} \mathrm{C}$ and $15 \mathrm{~min}$ of Taq polymerase activation at $95^{\circ} \mathrm{C}$, followed by 40 cycles of PCR at $95^{\circ} \mathrm{C}$ for $20 \mathrm{sec}$ (denaturation), $55^{\circ} \mathrm{C}$ for $30 \mathrm{sec}$ (annealing) and $72^{\circ} \mathrm{C}$ for $30 \mathrm{sec}$ (extension). Each experiment was conducted in duplicate.

Western blotting. Cells were washed with PBS and lysed with lysis buffer for $40 \mathrm{~min}$ on ice. Extracts were then centrifuged at $15,000 \times \mathrm{g}$ for $15 \mathrm{~min}$. Equal amounts of protein from the supernatant were electrophoresed on $8 \%$ SDS-polyacrylamide gel and sequentially electrophoretically transferred to a polyvinylidene difluoride (PVDF) membrane (Millipore, USA). After blocking with $5 \%$ non-fat milk in PBS containing $0.1 \%$ Tween-20 for $2 \mathrm{~h}$ at room temperature, membranes were incubated with monoclonal rabbit anti-Notch-1 antibody $(1: 5,000)$, anti-KLF4 antibody $(1: 1,000)$ and anti- $\beta$-actin (1:2,000; all by Abcam, USA) at $4^{\circ} \mathrm{C}$ overnight and washed three times for $5 \mathrm{~min}$ with PBS. Membranes were then incubated with horseradish peroxidase (HRP)-conjugated secondary goat anti-rabbit antibody $(1: 1,000)$ (Abcam) for $1 \mathrm{~h}$ at room temperature and washed three times for $5 \mathrm{~min}$ with PBS. Results were visualized by chemiluminescence using an enhanced chemiluminescence (ECL) immunoblotting kit (Cell Signaling Technology, USA) with a digital luminescent image analyzer LAS-1000 (Fujifilm, Tokyo, Japan). Band intensities were semi-quantified using densitometric analysis.

Statistical analysis. All data were analyzed with SPSS v. 18.0 (SPSS Inc., USA), and results are expressed as the means \pm standard deviation (SD). Two group comparisons were analyzed with a t-test, and $\mathrm{P}$-values were calculated. $\mathrm{P}<0.05$ was considered to indicate statistically significant differences.

\section{Results}

Transfection efficiency of siRNA. Fluorescence microscopy indicated that the Notch-1 expression plasmid psiRNA1 was successfully transfected into T24 and BIU87 cells. From 24 to $48 \mathrm{~h}$ after transfection, high levels of fluorescence were observed in transfected compared to non-transfected cells, which decreased by 72 h (Fig. 1). No significant differences in transfection efficiency were observed between T24 and BIU87 cells by fluorescence microscopy $(\mathrm{P}>0.05)$.

Notch-1 knockdown inhibits cell growth and induces apoptosis. MTT assay revealed that Notch-1 knockdown cells exhibited significantly lower proliferation rates at 24, 26, 48, 72 and 96 h compared to control cells $(\mathrm{P}<0.05)$ (Fig. 2A). No significant differences in growth rate were observed between Notch-1 knockdown T24 cells and Notch-1 knockdown BIU87 cells $(\mathrm{P}>0.05)$. Cell cycle status and apoptosis were detected at $72 \mathrm{~h}$ in T24 and BIU87 indicating mean apoptosis percentages of $13.75 \pm 1.23$ and $8.72 \pm 1.01$, respectively (Fig. 2B and C). Notch-1 knockdown significantly increased apoptosis in both 

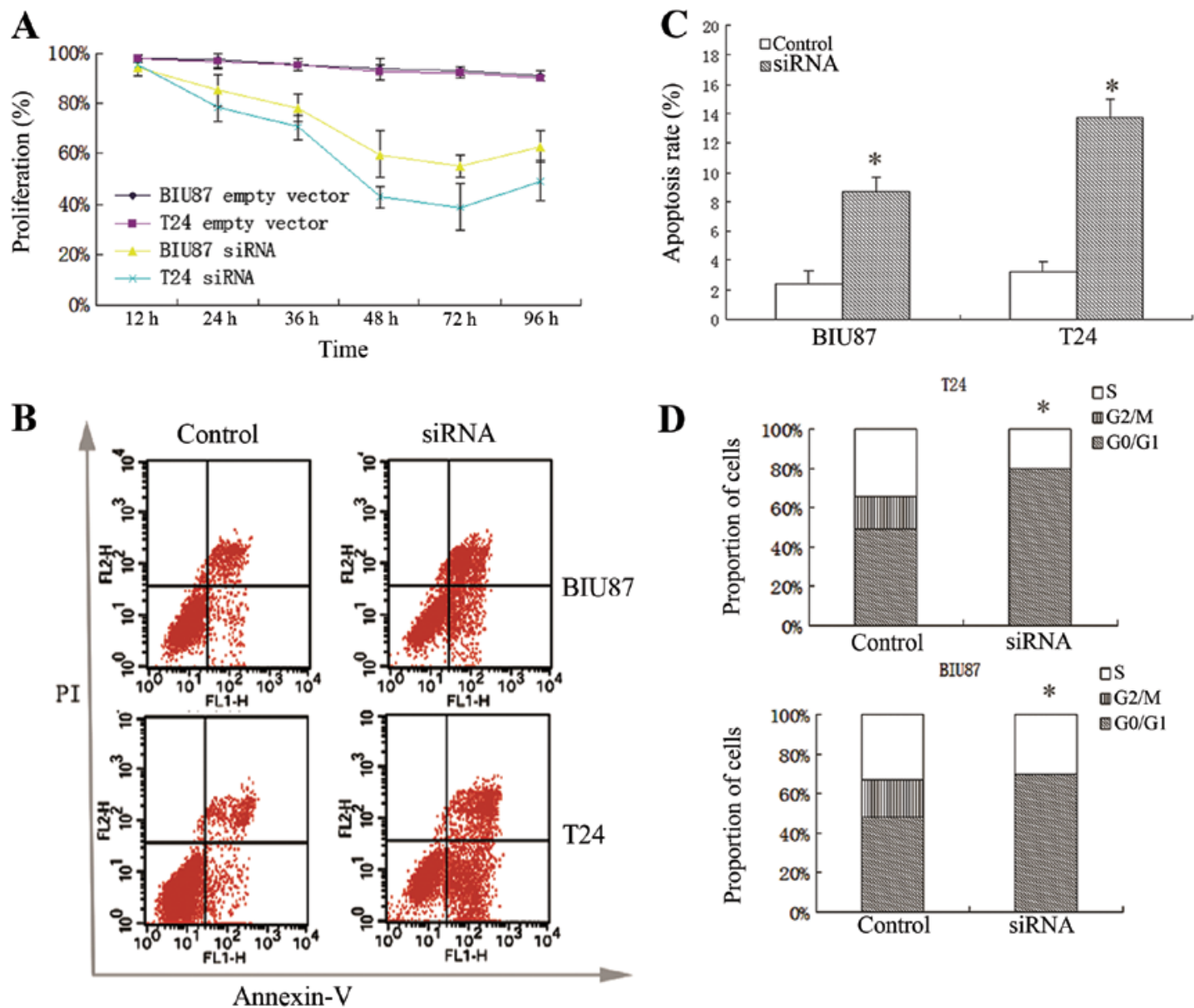

Figure 2. Downregulation of Notch-1 inhibits cell growth and induces apoptosis in transfected T24 and BIU87 cells. (A) MTT assay indicated that downregulation of Notch-1 inhibited cell proliferation and induced apoptosis. (B) Flow cytometry assay indicated that downregulation of Notch-1 induced apoptosis in T24 and BIU87 cells. (C) Apoptosis rate induced by downregulation of Notch-1 in T24 and BIU87 cells, ${ }^{*} \mathrm{P}<0.05$. (D) Flow cytometry indicated that transfected T24 and BIU87 cells exhibited G0/G1 arrest much more frequently than cells of the control groups, ${ }^{\text {"P }}<0.05$.

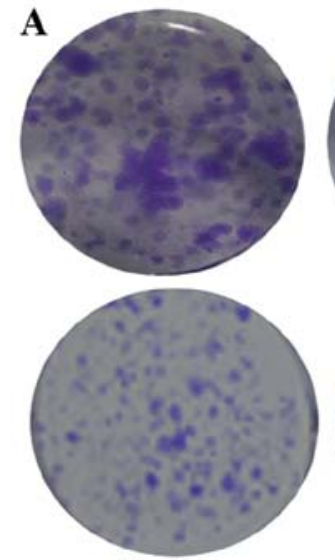

Control

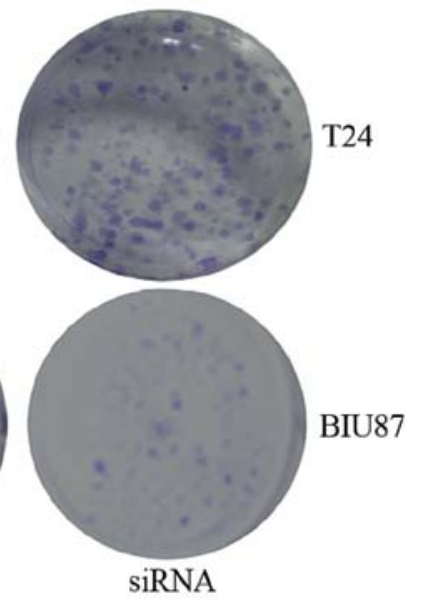

Figure 3. Cell viability is reduced in Notch-1 knockdown T24 and BIU87 cells. (A) Microscopic examination (x100) revealed that the number of colonies transfected with siRNA were significantly decreased compared to the control groups. (B) Reductions in colony number of cancer cells, including T24 and BIU87 cells, in Notch-1 knockdown cells transfected with siRNA compared to the control groups, ${ }^{\text {}} \mathrm{P}<0.05$.

cell lines $(\mathrm{P}<0.05)$. Furthermore, the proportion of cells in the G0/G1 phase (G0/G1 arrest) significantly increased after Notch-1 knockdown compared to those observed in the control groups $(\mathrm{P}<0.05$; Fig. 2D).
B

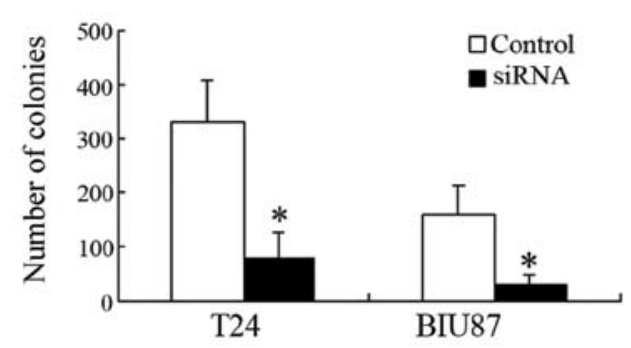

Notch-1 knockdown inhibits cell viability. Knockdown of Notch-1 inhibited cell viability in both cell lines, producing significantly fewer cancer cell colonies than observed in the control groups $(\mathrm{P}<0.05$; Fig. 3). 


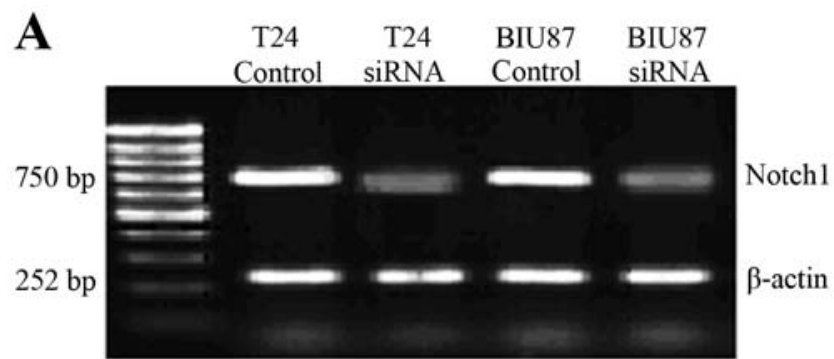

B

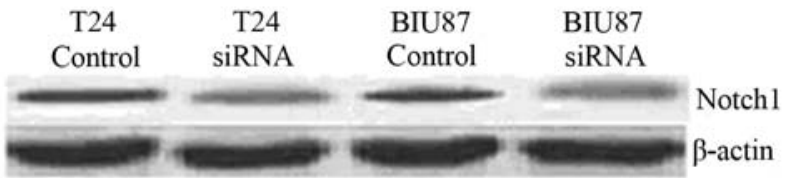

Figure 4. Introduction of psiRNA1 downregulates expression of Notch-1 mRNA and protein in both T24 and BIU87 cells. (A) Downregulation of Notch-1 mRNA expression examined in psiRNA1-transfected cells by semi-quantitative RT-PCR. (B) Effects of psiRNA1 downregulation on Notch-1 protein expression were examined in psiRNA1-transfected cells by western blot analysis.

A

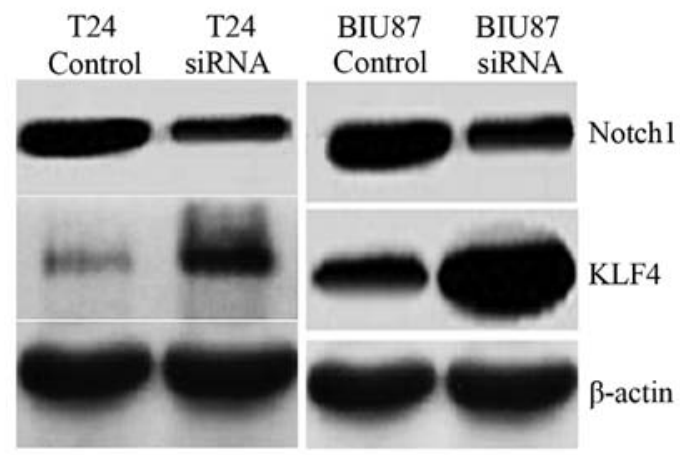

B

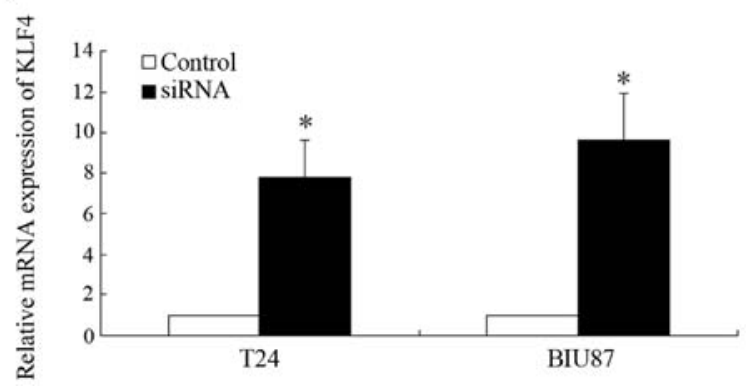

Figure 5. Notch-1 knockdown increases KLF4 expression. (A) KLF4 expression was significantly upregulated in Notch-1 knockdown T24 and BIU87 cells. (B) The mRNA expression of KLF4 was upregulated in Notch-1 knockdown T24 and BIU87 cells, ${ }^{*} \mathrm{P}<0.05$.

Notch-1 mRNA expression in Notch-1 knockdown cells. At 72 h, Notch-1 knockdown cells exhibited significantly lower Notch-1 mRNA expression in both T24 and BIU87 cells according to RT-PCR ( $\mathrm{P}<0.001$; Fig. 4A). Western blotting further confirmed that the expression of Notch-1 was significantly decreased by $72 \mathrm{~h}$ in both cell lines (Fig. 4B). KLF4 expression was significantly upregulated by Notch-1 knockdown (P<0.05; Fig. 5).

\section{Discussion}

By transfecting bladder cancer cell line T24 and BIU87 cultures with psiRNA1, the effects of Notch-1 knockdown and KLF4 knockdown were observed both independently and together, indicating that Notch-1 inhibits growth and apoptosis of bladder cancer cells. Both expression of Notch-1 mRNA and protein in T24 and BIU87 cells were decreased significantly following transfection, associated with Notch-1 downregulation. Furthermore, the mechanism of action was shown to be dependent on KLF4, suggesting that these two proteins are both important in bladder cancer tumorigenesis and metastasis. Thus, Notch-1 downregulation may provide a noteworthy target for inhibition of bladder cancer cell proliferation, with potential clinical implications.

The Notch signaling pathways have been implicated in the oncogenic processes involved in tumorigenesis, although conflicting data exist regarding the oncogenic and tumor suppressing role of Notch-1 (22). It is, however, possible that Notch-1 plays both roles, depending on specific cellular context and crosstalk with other signal-transduction pathways $(23,24)$. This may potentially explain the overexpression of Notch pathway components observed in human solid tumors, including those of uterine cervix cancer, endometrial cancer, head and neck squamous cell carcinomas, lung adenocarcinoma, renal cell carcinomas and neuroblastomas (24-29). In bladder cancer, Notch family expression patterns in papillary bladder transitional cell carcinoma have been reported to be very different from those observed in invasive bladder transitional cell carcinoma, suggesting that Notch-1 activity is a context-specific marker of prognosis and survival in these patients (30). Thus, aberrant Notch signaling in bladder cancer acts through complex and interdependent mechanisms that require further biochemical and clinical exploration.

Relationships between Notch-1 signaling and KLF4 have been previously reported. In colon cancer, inhibition of Notch-1 signaling was reported to reduce proliferation and tumor formation by increasing KLF4 expression (17,31). Since the human Notch-1 promoter is bound by KLF4, suppression of KLF4 in human breast cancer cells resulted in attenuation of Notch-1 expression (32). Furthermore, Ohnishi et al (19) reported that KLF4 expression was downregulated in bladder cancer cells and that expression of KLF4 inhibited cell growth and induced apoptosis (19). In the present study, consistent results were found, indicating that the expression of KLF4 mRNA and protein were significantly upregulated in T24 and BIU87 cells after Notch-1 knockdown. Additionally, proliferation of cells transfected with Notch-1 siRNA was significantly lower than cells downregulated of both Notch-1 and KLF4. Cumulatively, these findings suggest that Notch-1 regulates cell proliferation and differentiation in bladder cancer by directly inhibiting KLF4.

It is, however, important to consider the limitations of this model and the relationships that it suggests. While the upregulation and downregulation of KLF4 and Notch-1 may be indicative of a direct relationship, it is also possible that Notch-1 may signal through a distinct pathway in cells with increased KLF4 activity, as previously suggested (32). Further study is required to assess if multiple pathways exist and the potential crosstalk between these pathways, which may both 
be important in bladder cell tumorigenesis and metastasis. Additionally, future research should examine the anticancer effects of Notch-1 downregulation in vivo.

Notch-1 is important in bladder cancer cell proliferation and survival. Notch-1 may act as an oncogene, regulating the proliferation and differentiation of bladder cancer cells by inhibiting KLF4. Transfecting bladder cancer cells with psiRNA1 exerted significant effects on apoptosis and cell viability, and proliferation was inhibited by Notch-1 knockdown. Furthermore, the relationship between KLF4 and Notch-1 merits further consideration. While further study is required to elucidate the full mechanism of these pathways, this study provides a preliminary basis for the design of preclinical studies for possible therapeutic agents targeting the Notch-1 signaling pathway.

\section{References}

1. Kaufman DS, Shipley WU and Feldman AS: Bladder cancer. Lancet 374: 239-249, 2009.

2. Jemal A, Siegel R, Ward E, et al: Cancer statistics, 2008. CA Cancer J Clin 58: 71-96, 2008.

3. Urist MJ, Di Como CJ, Lu ML, et al: Loss of p63 expression is associated with tumor progression in bladder cancer. Am J Pathol 161: 1199-1206, 2002.

4. Junttila TT, Laato M, Vahlberg T, et al: Identification of patients with transitional cell carcinoma of the bladder overexpressing ErbB2, ErbB3, or specific ErbB4 isoforms: real-time reverse transcription-PCR analysis in estimation of ErbB receptor status from cancer patients. Clin Cancer Res 9: 5346-5357, 2003.

5. Habuchi T, Marberger M, Droller MJ, et al: Prognostic markers for bladder cancer: International Consensus Panel on bladder tumor markers. Urology 66: 64-74, 2005.

6. Shinohara $\mathrm{N}$ and Koyanagi T: Ras signal transduction in carcinogenesis and progression of bladder cancer: molecular target for treatment? Urol Res 30: 273-281, 2002.

7. Fletcher A, Choudhury A and Alam N: Metastatic bladder cancer: a review of current management. ISRN Urol 2011: 545241, 2011.

8. Hannon GJ: RNA interference. Nature 418: 244-251, 2002.

9. Paddison PJ and Hannon GJ: RNA interference: the new somatic cell genetics? Cancer Cell 2: 17-23, 2002.

10. Mummery-Widmer JL, Yamazaki M, Stoeger T, et al: Genomewide analysis of Notch signalling in Drosophila by transgenic RNAi. Nature 458: 987-992, 2009.

11. Krebs LT, Xue Y, Norton CR, et al: Notch signaling is essential for vascular morphogenesis in mice. Genes Dev 14: 1343-1352, 2000.

12. Brou C, Logeat F, Gupta N, et al: A novel proteolytic cleavage involved in Notch signaling: the role of the disintegrin-metalloprotease TACE. Mol Cell 5: 207-216, 2000.

13. Artavanis-Tsakonas S, Rand MD and Lake RJ: Notch signaling: cell fate control and signal integration in development. Science 284: 770-776, 1999.
14. Miele L and Osborne B: Arbiter of differentiation and death: Notch signaling meets apoptosis. J Cell Physiol 181: 393-409, 1999.

15. Hu YY, Zheng MH, Zhang R, Liang YM and Han H: Notch signaling pathway and cancer metastasis. Adv Exp Med Biol 727: 186-198, 2012.

16. Allenspach EJ, Maillard I, Aster JC and Pear WS: Notch signaling in cancer. Cancer Biol Ther 1: 466-476, 2002.

17. Ghaleb AM, Aggarwal G, Bialkowska AB, Nandan MO and Yang VW: Notch inhibits expression of the Krüppel-like factor 4 tumor suppressor in the intestinal epithelium. Mol Cancer Res 6: 1920-1927, 2008.

18. Bieker JJ: Krüppel-like factors: three fingers in many pies. J Biol Chem 276: 34355-34358, 2001.

19. Ohnishi S, Ohnami S, Laub F, et al: Downregulation and growth inhibitory effect of epithelial-type Krüppel-like transcription factor KLF4, but not KLF5, in bladder cancer. Biochem Biophys Res Commun 308: 251-256, 2003.

20. McConnell BB and Yang VW: Mammalian Krüppel-like factors in health and diseases. Physiol Rev 90: 1337-1381, 2010.

21. Ai X, Ju HZ and Wu ZH: The construction and identification of siRNA eukaryotic expression vector targeting Notch1 gene. J Clin Urol 5: 391-394, 2008.

22. Nicolas M, Wolfer A, Raj K, et al: Notch1 functions as a tumor suppressor in mouse skin. Nat Genet 33: 416-421, 2003.

23. Ehebauer M, Hayward P and Arias AM: Notch, a universal arbiter of cell fate decisions. Science 314: 1414-1415, 2006.

24. Radtke F and Raj K: The role of Notch in tumorigenesis: oncogene or tumour suppressor? Nat Rev Cancer 3: 756-767, 2003.

25. Wang L, Qin H, Chen B, Xin X, Li J and Han H: Overexpressed active Notch1 induces cell growth arrest of HeLa cervical carcinoma cells. Int J Gynecol Cancer 17: 1283-1292, 2007.

26. Suzuki T, Aoki D, Susumu N, Udagawa Y and Nozawa S: Imbalanced expression of TAN-1 and human Notch4 in endometrial cancers. Int J Oncol 17: 1131-1139, 2000.

27. Leethanakul C, Patel V, Gillespie J, et al: Distinct pattern of expression of differentiation and growth-related genes in squamous cell carcinomas of the head and neck revealed by the use of laser capture microdissection and cDNA arrays. Oncogene 19: 3220-3224, 2000.

28. Chen Y, De Marco MA, Graziani I, et al: Oxygen concentration determines the biological effects of Notch-1 signaling in adenocarcinoma of the lung. Cancer Res 67: 7954-7959, 2007.

29. Rae FK, Stephenson SA, Nicol DL and Clements JA: Novel association of a diverse range of genes with renal cell carcinoma as identified by differential display. Int J Cancer 88: 726-732, 2000.

30. van Limpt V, Chan A, Caron H, et al: SAGE analysis of neuroblastoma reveals a high expression of the human homologue of the Drosophila Delta gene. Med Pediatr Oncol 35: 554-558, 2000.

31. Shi TP, Xu H, Wei JF, et al: Association of low expression of notch-1 and jagged-1 in human papillary bladder cancer and shorter survival. J Urol 180: 361-366, 2008.

32. Zheng H, Pritchard DM, Yang X, et al: KLF4 gene expression is inhibited by the notch signaling pathway that controls goblet cell differentiation in mouse gastrointestinal tract. Am J Physiol Gastrointest Liver Physiol 296: G490-G498, 2009. 